\title{
Vancomycin MICs of the resistant mutants of $S$. aureus ATCC43300 vary based on the susceptibility test methods used
}

\author{
Yu-lin Zhu ${ }^{1}$, Qing Mei ${ }^{1}$, Li-fen $\mathrm{Hu}^{1}$, Jun Cheng ${ }^{1}$, Ying $\mathrm{Ye}^{1,2,3}$ and Jia-bin $\mathrm{Li}^{1,2,3}$
}

Clinical failures with vancomycin against meticillin-resistant Staphylococcus aureus (MRSA) infections have challenged vancomycin's standing as a first-line antimicrobial for these infections. Conventional MIC tests were not predictive of the in vivo therapeutic effect of vancomycin. Thus, we tested the susceptibility for the resistant mutants in the mutant selection window of S. aureus ATCC43300 (a MRSA strain) by three different MIC-testing methods in this paper. The MIC of vancomycin was estimated at $2 \mu \mathrm{g} \mathrm{ml}^{-1}$ on the Mueller-Hinton agar (MHA) plate only for the resistant mutant that was selected from the plate of vancomycin concentration $12 \mu \mathrm{g} \mathrm{ml}^{-1}$. The obvious changes of susceptibility testing were found between the resistant mutants and S. aureus ATCC43300 on the Brain-Heart Infusion Agar (BHIA) plates. There were subtle changes in the MIC trend within the susceptible range with the result of Etest for the resistant mutants. The susceptibility for the subcultures of resistant mutants would fall back when the external drug environment disappeared. In comparison with the $S$. aureus ATCC43300, sequence analysis revealed that there were no mutations in the staphylococcal protein A (spa) sequencing of the resistant mutants. The spa tape is $\mathbf{t} 421$ for all isolates.

The Journal of Antibiotics (2012) 65, 307-310; doi:10.1038/ja.2012.18; published online 11 April 2012

Keywords: MIC creep; MRSA; MSW; vancomycin; susceptibility testing

\section{INTRODUCTION}

Vancomycin has been largely used worldwide against meticillinresistant Staphylococcus aureus (MRSA) infections since the mid 1980s. ${ }^{1}$ As a result of empirical and directed therapy, clinicians have relied on only vancomycin for many years in the management of serious MRSA infections and have enjoyed a substantial period with no vancomycin resistance appearing in $S$. aureus. ${ }^{2,3}$ However, clinical failures with vancomycin against MRSA infections have challenged vancomycin's standing as a first-line antimicrobial for these infections. MIC creep may be one of the reasons for increasing vancomycin failure rates, which have been reported for MRSA strains with vancomycin MIC values of $\geqslant 1 \mu \mathrm{g} \mathrm{ml}^{-1}$ in patients with MRSA bacteremia and/or pneumonia. ${ }^{4,5}$ Recent studies have shown that these MIC increases for vancomycin were not reliably detected by percentage susceptibility as occurred below the susceptibility breakpoint and could affect the clinical efficacy. ${ }^{6,7}$ However, few studies gave the reasons for the MIC creep phenomenon.

Zhao and Drlica ${ }^{8,9}$ first put forward the mutant selection window (MSW) hypothesis. This hypothesis maintains that drug-resistant mutant subpopulations present prior to initiation of antimicrobial treatment are enriched and amplified during therapy when antimicrobial concentrations fall within a specific range (the
MSW). ${ }^{10}$ The upper boundary of the MSW is the MIC of the least drug-susceptible mutant subpopulation, a value called the mutant prevention concentration (MPC). ${ }^{11}$ The lower boundary of the MSW is the lowest concentration that exerts selective pressure, often approximated by the minimal concentration that inhibits colony formation by $99 \%\left(\mathrm{MIC}_{99}\right)$. According to this hypothesis, the possible reason for clinical failures was the concentration of drugs in vivo falling within the MSW so that resistant mutants were enriched, with a concomitant loss in susceptibility.

As to vancomycin, the loss in susceptibility in MSW may be the reason for the MIC creep. Conventional MIC tests were not predictive of the in vivo therapeutic effect of vancomycin. ${ }^{12}$ Unless appropriate testing methods are used and the data carefully analyzed, such subtle changes in the MIC trend within the susceptible range (MIC creep) would be missed. ${ }^{13}$ It has been reported that vancomycin MICs generated by Etest were consistently one twofold dilution higher than those determined by Clinical and Laboratory Standards Institute (CLSI) broth dilution method. ${ }^{14,15}$ In addition, the BHIA plates being recommended to screen the $S$. aureus reduced susceptibility to vancomycin in the CLSI. ${ }^{16}$ Thus, we tested the susceptibility of the resistant mutants of S. aureus ATCC43300 by three different MIC-testing methods in this paper. The study

${ }^{1}$ Department of Infectious Diseases, The First Affiliated Hospital of Anhui Medical University, Hefei, Anhui, China; ${ }^{2}$ Anhui Center for Surveillance of Bacterial Resistance, Hefei, Anhui, China and ${ }^{3}$ Institute of Bacterium Resistance, Anhui Medical University, Hefei, Anhui, China

Correspondence: Professor Y Ye or Professor J-b Li, Department of Infectious Diseases, The First Affiliated Hospital of Anhui Medical University, Hefei, Anhui 230022, China. E-mail: gao3ye2@yahoo.com.cn or lijiabin948@vip.sohu.com

Received 14 November 2011; revised 28 February 2012; accepted 29 February 2012; published online 11 April 2012 
described here was to explain the relationship between the MIC creep and the MSW for vancomycin.

\section{RESULTS}

The MIC, $\mathrm{MIC}_{99}$ and MPC of vancomycin for S. aureus ATCC43300 The MIC and $\mathrm{MIC}_{99}$ of vancomycin were estimated at $1 \mu \mathrm{g} \mathrm{ml}^{-1}$ and $0.8 \mu \mathrm{g} \mathrm{ml}^{-1}$, respectively, for S. aureus ATCC43300. The MPC of vancomycin was estimated at $16 \mu \mathrm{g} \mathrm{ml}^{-1}$ by agar double-dilution method. The exact MPC of vancomycin was estimated at $14.4 \mu \mathrm{g} \mathrm{ml}^{-1}(16 \times 90 \%)$ for S. aureus ATCC43300. All experiments were performed in duplicate.

\section{Susceptibility testing on the MHA and BHIA plates}

According to the MSW hypothesis, the resistant mutants were selected from different antibiotic concentrations in the MSW $(1,2,4,8,12$ and $14 \mu \mathrm{g} \mathrm{ml}^{-1}$ ). The MIC of vancomycin was estimated at $2 \mu \mathrm{g} \mathrm{ml}^{-1}$ on the MHA plate only for the resistant mutant that was selected from the plate of vancomycin concentration $12 \mu \mathrm{g} \mathrm{ml}^{-1}$. However, the MICs of vancomycin were estimated at $1 \mu \mathrm{g} \mathrm{ml}^{-1}$ on the MHA plates for the other resistant mutants, which was the same as $S$. aureus ATCC43300. The obvious changes of susceptibility testing were found between the resistant mutants and S. aureus ATCC43300 on the BHIA plates. The results of the susceptibility testing for resistant mutants and $S$. aureus ATCC43300 are shown in Table 1.

\section{Table 1 Susceptibility testing for resistant mutants on MHA and BHIA plates}

\begin{tabular}{lcc}
\hline Strains $^{\mathrm{a}}$ & MHA $\left(\mu \mathrm{gml^{-1 }}\right)$ & ${\text { BHIA }\left(\mu g \mathrm{I}^{-1}\right)}^{-1}$ \\
\hline Staphylococcus aureus ATCC43300 & 1 & 2 \\
The resistant mutant 1 & 1 & 2 \\
The resistant mutant 2 & 1 & 4 \\
The resistant mutant 4 & 1 & 4 \\
The resistant mutant 8 & 1 & 4 \\
The resistant mutant 12 & 2 & 8 \\
The resistant mutant 14 & 1 & 4
\end{tabular}

aThe resistant mutants $1,2,4,8,12$ and 14 mean the resistant mutants selected from the plates of the vancomycin concentrations of $1,2,4,8,12$ and $14 \mu \mathrm{g} \mathrm{ml}^{-1}$, respectively.

Table 2 Etest for the resistant mutants and their subcultures

\begin{tabular}{lcccc}
\hline & $\begin{array}{c}\text { The original } \\
\text { strains } \\
\left(\mu g m l^{-1}\right)\end{array}$ & $\begin{array}{c}\text { First }^{b} \\
\left(\mu g \mathrm{ml}^{-1}\right)\end{array}$ & $\begin{array}{c}\text { Second }^{b} \\
\left(\mu g \mathrm{ml}^{-1}\right)\end{array}$ & $\begin{array}{c}\text { Third }^{b} \\
\left(\mu g \mathrm{ml}^{-1}\right)\end{array}$ \\
$\begin{array}{l}\text { Strains } \\
\text { a }\end{array}$ & 1 & 1 & 1 & 1 \\
\hline $\begin{array}{l}\text { Staphylococcus aureus } \\
\text { ATCC43300 }\end{array}$ & 1.5 & 1.5 & 1 & 1 \\
The resistant mutant 1 & 1.5 & 1.5 & 1 & 1 \\
The resistant mutant 2 & 1.5 & 1.5 & 1 & 1 \\
The resistant mutant 4 & 1.5 & 1.5 & 1 & 1 \\
The resistant mutant 8 & 2 & 2 & 1.5 & 1 \\
The resistant mutant 12 & 1.5 & 1.5 & 1 & 1 \\
The resistant mutant 14 & $14 \mathrm{~m}$ & & 1 \\
\hline
\end{tabular}

aThe resistant mutants $1,2,4,8,12$ and 14 mean the resistant mutants selected from the plates of the vancomycin concentrations of $1,2,4,8,12$ and $14 \mu \mathrm{g} \mathrm{ml}^{-1}$, respectively. birst, second and third mean the first passage culture, the second passage culture and the third passage culture.
Etest for the resistant mutants and their subcultures

The subtle changes in the MIC trend within the susceptible range were found by the Etest when the concentration of drugs fell in the MSW (Table 2). The MIC of vancomycin was estimated at $2 \mu \mathrm{g} \mathrm{ml}^{-1}$ for the resistant mutant that was selected from the plate of vancomycin concentration of $12 \mu \mathrm{g} \mathrm{ml}^{-1}$ and on their third passage, the culture fell back to $1 \mu \mathrm{g} \mathrm{ml}^{-1}$. However, the MICs of vancomycin were estimated at $1.5 \mu \mathrm{g} \mathrm{ml}^{-1}$ for the other resistant mutants and on their second passage, the culture fell back to $1 \mu \mathrm{g} \mathrm{ml}^{-1}$.

\section{Spa type and molecular analysis}

All isolates of $S$. aureus ATCC43300 and the resistant mutants analyzed were typeable by spa sequencing. In comparison with the $S$. aureus ATCC43300, sequence analysis revealed that there were no mutations in the spa sequencing of the resistant mutants. The spa repeat profile is WGKAOM for all isolates. The name of the spa tape is $\mathrm{t} 421$ according to the Ridom SpaServer-SpaTypes (http://spa. ridom.de/spatypes.shtml).

\section{DISCUSSION}

Methicillin-resistant S. aureus (MRSA) is a major public health threat. For decades, glycopeptides have been the treatment of choice for serious MRSA infections such as bacteremia and ventilator-associated pneumonia. Thus, the association of vancomycin-treatment failures with increased vancomycin MICs is a concern, especially as these MICs are within what is considered the susceptible range. ${ }^{17,18}$ Studies reporting vancomycin MIC changes over time among MRSA have shown conflicting results. Several factors have been put forward to explain the discrepant findings, including the method of analysis, clonal dissemination and intensity of vancomycin usage. ${ }^{19-21}$ The perception of MIC creep may vary according to the methods used to analyze the data. Vancomycin MICs did not creep in S. aureus isolates from 2002 to 2006 in a setting with low vancomycin usage when MICs for the different groups in the different years were compared with the linear-trend $X^{2}$ test. $^{19}$ However, small vancomycin MIC creeps were found at three centers of nine US medical centers when data were analyzed by the geometric mean method. ${ }^{20}$ This increase in MICs over time has been referred to as 'MIC creep', which occurs when MIC values increase but are not detected because the percentage of susceptible isolates remains relatively unchanged. Thus, vancomycin MIC creep should be recognized as a gradual increase in the central tendency of the vancomycin MIC for the dominant wild-type population, and it is caused by long-term and/or extensive use of vancomycin. These increases may portend development of resistance and contribute to the diminishing effectiveness of vancomycin in serious MRSA infections.

Antimicrobial resistance is a complex problem that may require attention at many levels. ${ }^{22}$ Issues concerning dosing are addressed by the MSW hypothesis. ${ }^{8,9}$ The study at the beginning depended heavily on fluoroquinolone action and resistance to support the selection window concept. ${ }^{11}$ Then, the definition of the boundaries of the window was expanded to other antimicrobial agents, such as chloramphenicol, tobramycin, rifampicin, vancomycin and penicillin, by using $S$. aureus. ${ }^{8}$ A broad plateau in mutant recovery is observed for vancomycin with $S$. aureus ATCC43300 just like the fluoroquinolones with mycobacteria in the study. Perhaps it is the decisive reason for the treatment failures. Vancomycin is a concentrationindependent antibiotic (also referred to as a 'time-dependent' antibiotic), and there are factors that affect its clinical activity, including variable tissue distribution, inoculum size and emerging resistance. ${ }^{24}$ Peak serum levels with a dose of $1 \mathrm{~g}$ every $12 \mathrm{~h}$ are usually 
between 25 and $40 \mu \mathrm{g} \mathrm{ml}^{-124}$, which is higher than the average MPC of vancomycin for most clinical MRSA, but in some tissue, such as lung infection, it may fall in MSW so that resistant mutants were enriched, with a concomitant loss in susceptibility. So vancomycin MIC creeps were found over time for long-term and/or extensive use of vancomycin.

Compared with the $S$. aureus ATCC43300, only the resistant mutant that was selected from the plate of vancomycin concentration of $12 \mu \mathrm{g} \mathrm{ml}^{-1}$ had higher MIC of vancomycin on the MHA plates. The vancomycin MICs increased on MHA plates for other resistant mutants were not reliably detected by percentage susceptibility as they occurred below the susceptibility breakpoint. These subtle changes in the MIC trend within the susceptible range would be found by the Etest (Table 2). However, more obvious changes were found on the BHIA plates. The reason may be the Brilliant nutrient BHI media can promote the growth of MRSA, especially hetero vancomycin-resistant staphylococcus (VRS). ${ }^{12}$ The highest MICs of vancomycin for the resistant mutants that were selected from the plates of vancomycin concentration $12 \mu \mathrm{g} \mathrm{ml}^{-1}$ (upper portions of the window) were both found on the MHA plates and BHIA plates. Similar phenomenon was found with quinolone susceptibility and identity of resistant mutants selected in different portions of the window. ${ }^{25}$ Testing involving $S$. aureus for vancomycin and using in vitro dynamic models revealed that mutants are selectively amplified when concentrations are between the MIC and the MPC. ${ }^{26}$ Stepwise accumulation of drug resistance on the MSW was examined in fluoroquinolone resistance of Haemophilus influenzae. ${ }^{27}$ Observations strongly suggested that these vancomycin-intermediate $S$. aureus isolates represent the progeny of a single MRSA strain that emerged in vivo under the selective pressure of vancomycin therapy. ${ }^{28}$ Even though there is no evidence of MRSA in vivo tests for the MSW of vancomycin, this kind of phenomena and their consequences required concern and took measures.

The problem of antibiotic resistance has been worsening because the patient population having weakened immune systems has been increasing from factors such as aging, radiotherapy, infection with HIV and treatment with immunosuppressive chemicals ${ }^{8}$. The susceptibility for the subcultures of resistant mutants would fall back when the external drug environment disappeared. If the changing vancomycin phenotypes were the products of selective antibiotic pressure in vivo, then it might be possible to reconstruct these phenotypic changes without the pressure. However, the clinical and microbiological response for the infection sites may vary depending on vancomycin penetration to the infection site and the immune-system response. ${ }^{29}$ Vancomycin penetrates into most body spaces, although the concentrations obtained are variable and somewhat dependent on the degree of inflammation present. ${ }^{23}$ As to the complicated infections for patient population having weakened immune systems, resistant mutants must be generated, and then those mutants must be selectively enriched (selected) in the bacterial population under the therapeutic concentrations currently used for a long time. To sum up, optimizing vancomycin dosing according to the pharmacokinetic-pharmacodynamic principles has the potential to maximize the efficacy of vancomycin against MRSA isolates.

\section{METHODS}

\section{Antimicrobial agents, bacterial strain and susceptibility testing}

Vancomycin was provided by Eli Lilly Japan K.K, Seishin Laboratories (Kobe, Japan). S. aureus ATCC43300 was stored at the Anhui Center for Surveillance of Bacterial Resistance. MICs of antimicrobial agents were determined by means of the agar-dilution method according to a standard procedure described by the CLSI in 2010. ${ }^{16}$ The measurement was followed by a second determination, plus a replicate that utilized linear drug concentration increments $(10 \%$ per sequential increase). The fraction of the colonies recovered was plotted against the drug concentration to determine $\mathrm{MIC}_{99}$ by interpolation.

\section{MPC determination}

The MPCs were determined as described elsewhere. ${ }^{9}$ Briefly, the tested bacteria were cultured in Mueller-Hinton broth and incubated for $24 \mathrm{~h}$. Then, the suspension was centrifuged ( $4000 \mathrm{~g}$ for $10 \mathrm{~min}$ ) and resuspended in MuellerHinton broth to yield a concentration of $3 \times 10^{11} \mathrm{cfu} \mathrm{ml}^{-1}$. The MPC was defined as the lowest drug concentration that prevented bacterial colony formation from a culture containing $>10^{10}$ bacteria. The inoculated plates were incubated for $72 \mathrm{~h}$ at $35^{\circ} \mathrm{C}$ and screened visually for growth. To estimate the MPC, logarithms of bacterial numbers were plotted against antibiotic concentrations (Figure 1). MPC was taken as the point where the plot intersected the theoretical limit of detection $\left(\log \mathrm{cfu} \mathrm{ml}^{-1}=1\right)$.

\section{Susceptibility testing for resistant mutants}

The resistant mutants were selected from different antibiotic concentrations in the MSW (lower, middle and upper portions of the window). The resistant mutants were cultured in Mueller-Hinton broth and incubated for $24 \mathrm{~h}$. Then, the suspension was centrifuged $(4000 \mathrm{~g}$ for $10 \mathrm{~min}$ ) and diluted with sterile saline $(0.9 \% \mathrm{NaCl})$. Bacterial liquid concentration was first adjusted to 0.5McFarland-standard (about $1.5 \times 10^{8} \mathrm{cfu} \mathrm{ml}^{-1}$ ). Then, the bacterial liquid was diluted with sterile saline to $10^{6} \mathrm{cfu} \mathrm{ml}^{-1}$. The plates were supplemented with vancomycin concentrations in the range of MIC to MPC (double dilution). The MHA and BHIA plates were inoculated with $10 \mu \mathrm{l}$ of a suspension of the testing isolate equivalent to $10^{4} \mathrm{cfu} \mathrm{ml}^{-1}$. Test plates were incubated in ambient air at $35^{\circ} \mathrm{C}$ and were observed after $24 \mathrm{~h}$. S. aureus ATCC43300 was tested on each plate at the same time.

\section{Etest for the resistant mutants}

MIC testing for the resistant mutants and S. aureus ATCC43300 was performed using the Etest method, following the manufacturer's guidelines. The vancomycin Etest strips (AB bioMerieux, Solna city, Sweden) were applied to the agar surface using an Etest applicator and were not moved following application. MICs were read by a single observer in accordance with the manufacturer's guidelines. The resistant mutants were serially subcultured on vancomycin-free MHA for three times and susceptibility testing was done for them by the vancomycin Etest strips.

\section{Staphylococcal protein A (spa) typing and molecular analysis}

Spa is a major surface protein found in essentially all strains of each $S$. aureus. The spa typing was performed on each of S. aureus ATCC43300 and the resistant mutants as previously described. ${ }^{30}$ Primer sites for PCR amplification were designed according to the published sequence data. A negative control (sterile deionized water) and a positive control (S. aureus collection from our laboratory) were included. Purified PCR products were ligated with the

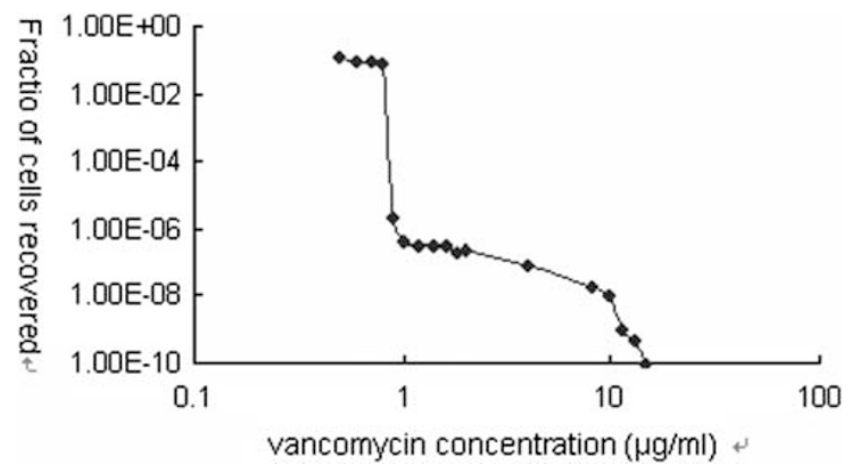

Figure 1 Effect of vancomycin concentrations on selection of resistant mutants. A full color version of this figure is available at The Journal of Antibiotics journal online. 
pMD18-T easy vector (TaKaRa, Dalian, China) and expressed in Escherichia coli JM109 on a Luria-Bertani agar plate with blue-white selection. Nucleotide sequences were determined three times by bidirectional sequencing of PCR production with a 3730 automatic DNA sequencer (Applied Biosystems, Foster City, CA, USA).

\section{CONFLICT OF INTEREST}

The authors declare no conflict of interest.

\section{ACKNOWLEDGEMENTS}

This work was supported by the National Natural Science Foundation of China (No. 81071394).

1 Kirst, H. A., Thompson, D. G. \& Nicas, T. I. Historical yearly usage of vancomycin Antimicrob. Agents Chemother. 42, 1303-1304 (1998).

2 Hiramatsu, K. et al. Methicillin-resistant Staphylococcus aureus clinical strain with reduced vancomycin susceptibility. J. Antimicrob. Chemother. 40, 135-136 (1997)

3 Centers for Disease Control and Prevention. Staphylococcus aureus resistant to vancomycin, United States, 2002. Morb. Mortal. Weekly Rep. 51, 565-567 (2002).

4 Lodise, T. P., Miller, C. D. \& Graves, J. et al. Predictors of high vancomycin MIC values among patients with methicillin-resistant Staphylococcus aureus bacteraemia. J. Antimicrob. Chemother. 62, 1138-1141 (2008).

5 Lodise, T. P. et al. Relationship between vancomycin MIC and failure among patients with methicillin-resistant Staphylococcus aureus bacteremia treated with vancomycin. Antimicrob. Agents Chemother. 52, 3315-3320 (2008).

6 Steinkraus, G., White, R. \& Friedrich, L. Vancomycin MIC creep in non-vancomycinintermediate Staphylococcus aureus (VISA), vancomycin-susceptible clinical methicillin-resistant S. aureus (MRSA) blood isolates from 2001-05. J. Antimicrob. Chemother. 60, 788-794 (2007).

7 Gould, I. M. Clinical relevance of increasing glycopeptide MICs against Staphylococcus aureus. Inter. J. Antimicrob. Agents. 31, 1-9 (2008).

8 Zhao, X. \& Drlica, K. Restricting the selection of antibiotic-resistant mutants: a genera strategy derived from fluoroquinolone studies. Clin. Infect. Dis. 33(Suppl 3), S147-S156 (2001).

9 Zhao, X. \& Drlica, K. Restricting the selection of antibiotic-resistant mutants: measurement and potential uses of the mutant selection window. J. Infect. Dis. 185, 561-565 (2002).

10 Drlica, K. \& Zhao, X. Mutant selection window hypothesis updated. Clin. Infect. Dis. 44, 681-688 (2007)

11 Dong, Y. et al. Effect of fluoroquinolone concentration on selection of resistant mutants of Mycobacterium bovis BCG and Staphylococcus aureus. Antimicrob. Agents Chemother. 43, 1756-1758 (1999).

$12 \mathrm{Wu}, \mathrm{B}$. et al. Staphylococcus heterogeneously resistant to vancomycin in China and antimicrobial activities of imipenem and vancomycin in combination against it. J. Clin. Microbiol. 40, 1109-1112 (2002).
13 Ho, P. L. et al. Vancomycin MIC creep in MRSA isolates from 1997 to 2008 in a healthcare region in Hong Kong. J. Infec. 60, 140-145 (2010).

14 Prakash, V., Lewis, II J. S. \& Jorgensen, J. H. Vancomycin MICs for methicillin-resistant Staphylococcus aureus isolates differ based upon the susceptibility test method used. Antimicrob. Agents Chemother. 52, 4528 (2008).

15 Mason, E. O. et al. Vancomycin MICs for Staphylococcus aureus vary by detection method and have subtly increased in a pediatric population since 2005. J. Clin. Microbiol. 47, 1628-1630 (2009).

16 Clinical and Laboratory Standards Institute. NCCLS performance standards for antimicrobial susceptibility testing; Twentieth Informational Supplement CLSI/NCCLS document M100-S20. Techstreet 30, 1-160 (2010).

17 Moise-Broder, P. A. et al. Accessory gene regulator group II polymorphism in methicillin-resistant Staphylococcus aureus is predictive of failure of vancomycin therapy. Clin. Infect. Dis. 38, 1700-1705 (2004).

18 Sakoulas, G. et al. Relationship of MIC and bactericidal activity to efficacy of vancomycin for treatment of methicillin-resistant Staphylococcus aureus bacteremia. J. Clin. Microbiol. 42, 2398-2402 (2004).

19 Alos, J. I. et al. Vancomycin MICs did not creep in Staphylococcus aureus isolates from 2002 to 2006 in a setting with low vancomycin usage. J. Antimicrob. Chemother. 62 773-775 (2008).

20 Sader, H. S. et al. Evaluation of vancomycin and daptomycin potency trends (MIC creep) against methicillin-resistant Staphylococcus aureus isolates collected in nine U.S. medical centers from 2002 to 2006. Antimicrob. Agents Chemother. 53, 4127-4132 (2009).

21 Moise, P. A. et al. Susceptibility relationship between vancomycin and daptomycin in Staphylococcus aureus: facts and assumptions. Lancet Infect. Dis. 9, 617-624 (2009)

22 Levy, S. B. \& Marshall, B. Antibacterial resistance worldwide: causes, challenges, and responses. Nat. Med. 10, S122-S129 (2004).

23 Rybak, M. J. The pharmacokinetic and pharmacodynamic properties of vancomycin Clin. Infect. Dis. 42, S35-S39 (2006).

24 Kucers, A. et al. The Use of Antibiotics: a Clinical Review of Antibacterial, Antifungal, and Antiviral drugs. 5th ed. (Butterworth-Heinemann, BostonMass, 1997).

25 Cui, J. et al. The mutant selection window demonstrated in rabbits infected with Staphylococcus aureus. J. Infect. Dis. 194, 1601-1608 (2006).

26 Firsov, A. A. et al. Testing the mutant selection window hypothesis with Staphylococcus aureus exposed to daptomycin and vancomycin in an in vitro dynamic model. J. Antimicrob. Chemother. 58, 1185-1192 (2006).

$27 \mathrm{Li}, \mathrm{X}$. et al. Quinolone-resistant Haemophilus influenzae: determination of mutan selection window for ciprofloxacin, garenoxacin, levofloxacin, and moxifloxacin. Antimicrob. Agents Chemother. 48, 4460-4462 (2004).

28 Sieradzki, K. et al. Evolution of a vancomycin-intermediate Staphylococcus aureus strain in vivo: Multiple changes in the antibiotic resistance phenotypes of a single lineage of methicillin-resistant $S$. aureus under the impact of antibiotics administered for chemotherapy. J. Clin. Microbiol. 41, 1687-1693 (2003).

29 Giuliano, C. et al. Use of vancomycin pharmacokinetic-pharmacodynamic properties in the treatment of MRSA infections. Expert Rev. Anti. Infect. Ther. 8 95-106 (2010).

30 Shopsin, B. et al. Evaluation of protein A gene polymorphic region DNA sequencing for typing of Staphylococcus aureus strains. J. Clin. Microbiol. 37, 3556-3563 (1999). 